\title{
Kritische Bemerkungen zum Nationalen Forschungsprogramm NFP53
}

\author{
Für den Autor des folgenden Beitrags brachte das NFP53 «Muskuloskelettale Gesund- \\ heit - chronische Schmerzen» keine grundlegend neuen Erkenntnisse. Er kritisiert \\ unter anderem, dass auf die Mitarbeit von Medizinern, die im Bereich des Bewegungs- \\ apparates tätig sind, weitgehend verzichtet wurde, auch die Fachliteratur sei zu \\ wenig berücksichtigt worden. War es ein vorwiegend politisch motiviertes \\ Forschungsprojekt und eigentlich überflüssig?
}

Das Studium der Broschüre des Schweizerischen Nationalfonds SNF über die Ergebnisse aus 5 Jahren Forschung im Rahmen des NFP53 «Muskuloskelettale Gesundheit - chronische Schmerzen» [1] und die Durchsicht des Sammelbandes der im Schweizerischen Medizin-Forum erschienenen Publikationen (Sonderdruck der Editores Medicorum Helveticorum EMH 2010) muss bei den meisten gutausgebildeten und praxiserfahrenen orthopädischen Chirurgen Erstaunen und kritische Bemerkungen provozieren.

\section{Begründung und Entstehung des NFP53}

Erkrankungen des Bewegungsapparates verursachen gemäss den Angaben in der Broschüre jährlich betriebliche Kosten von 0,97 Milliarden Franken und durch Produktionsverluste 3,3 Milliarden Franken. Laut Statistik der Invalidenversicherung wird 1/5 der Renten wegen Erkrankungen des Bewegungsapparates, besonders wegen chronischer Rückenbeschwerden, gesprochen, was jährlich Kosten von 1 Milliarde Franken verursacht.

Da die muskuloskelettalen Erkrankungen lange Zeit von der Forschung vernachlässigt worden seien (eine nicht zutreffende, geradezu groteske Behauptung), bestehe das Ziel des NFP53 darin, die Frage zu klären, wie die Forschung dazu beitragen könne, die muskuloskelettale Gesundheit der Menschen in der Schweiz aufrechtzuerhalten oder wiederherzustellen.

Im Januar 2003 beschloss der Bundesrat, Erkrankungen des Bewegungsappartes besser zu erforschen wegen ihrer grossen Bedeutung für die Volksgesundheit und die Gesundheitsökonomie. Mit der Durchführung des Programms wurde der Schweizerische Nationalfonds SNF beauftragt. Von den 71 Forschungsgesuchen für konkrete Forschungsprojekte bewilligte der Forschungsrat der Abteilung I.V. des SNF schliesslich 26 Projekte, die mehrheitlich interdisziplinär angelegt waren mit Forschern aus den unterschiedlichs- ten Fachgebieten: naturwissenschaftliche Grundlagendisziplinen, medizinische Fachbereiche, technische Wissenschaften, Epidemiologie, Versicherungsmedizin, Statistik und Ökonomie.

\section{Angeblich neue Erkenntnisse des NFP53}

Die Broschüre erwähnt bekannte Risikofaktoren für die Coxarthrose als neu, kritisiert die üblichen Behandlungsformen bei Rückenbeschwerden als zu wenig auf eine rasche und dauerhafte Rückkehr an den Arbeitsplatz ausgerichtet und kritisiert Unternehmen wegen Mängeln in der frühzeitigen Erfassung von Mitarbeitern mit Rückenschmerzen.

Als weitere Ergebnisse werden die Förderung der Selbsthilfe bei chronischen Rückenschmerzen und die Erkenntnis der Nichtwirksamkeit von Chondroitin gegen Arthrose erwähnt. Ferner wird als neues Ergebnis zitiert, dass Patienten mit chronischen Rückenschmerzen aktiv und funktionell orientiert behandelt werden. Zur Prävention von Stürzen und Frakturen bei älteren Menschen wird Physiotherapie und Vitamin-D-Zufuhr empfohlen. Erwähnt werden bekannte regionale Unterschiede in der medizinischen Versorgung von Affektionen des Bewegungsapparates und die Gefahr der Überbehandlung

Aus den «wegweisenden Resultaten» des NFP53, welche die Prävention und die Behandlung von Erkrankungen des Bewegungsapparates voranbringen sollten, wurden Empfehlungen an den Bundesrat formuliert. Ihm wurde übertragen, über konkrete Massnahmen zu befinden, die einem gesunden Bewegungsapparat dienlich sein können.

\section{Kritik des NFP aus orthopädisch-chirurgischer Sicht}

Ein gutausgebildeter und praktisch erfahrener orthopädischer Chirurg nimmt Anstoss an der seltsamen Bezeichnung des NFP53 als «Muskuloskelettale 
Gesundheit - chronische Schmerzen», handelt es sich doch um den grossen medizinischen Bereich des Bewegungsapparates, der nicht nur aus dem Skelett und Muskeln, sondern auch aus Gelenkkapseln, Bändern, Gefässen und Nerven besteht. Ferner sind andauernde (chronische) Schmerzen und Beschwerden nicht charakteristisch für den Bewegungsapparat. Denn üblicherweise kommen und gehen die Beschwerden und Schmerzen im Rücken und in arthrotischen Gelenken, können längere Zeit verschwinden und sogar in Händen, Füssen und im Knie mit der Zeit geringer werden, aber auch bei rascher Progredienz, besonders im Hüftgelenk, eine operative Therapie notwendig machen. Die Spontanremissionstendenz ist bei Beschwerden und Schmerzen im Rücken, bei Arthrosen und Sehnenansätzen sehr gross [2, 3, 4, $5,6,7,8]$. die in entwickelten Ländern bisher für den menschlichen Bewegungsapparat als zuständig betrachtet wurden und recht erfolgreich zusammengearbeitet haben: Orthopädische Chirurgen, Anatome, Pathologen,Radiologen, Allgemeinpraktiker, Neurologen, Neurochirurgen, Rheumatologen, Psychiater und Physiotherapeutinnen.

Des weiteren muss Anstoss genommen werden am Ausserachtlassen der umfangreichen Literatur über den Bewegungsapparat. Es existiert eine umfangreiche Literatur über die Pathologie der Wirbelsäule [9 und andere Zeitschriften] und über die Problematik der Rückenbeschwerden für Patienten, Arbeitgeber und das Versicherungswesen [3, 10]. Der Behandlungseifer bei harmlosen Haltungsvarianten im Kindesalter im Bereich des Rückens, der Knie und Füsse ist kontraproduktiv [11]. Auffallend ist der Um-

\section{Bei Rückenbeschwerden steht die Tendenz zur spontanen Remission mit beschwerdefreien Intervallen im Widerspruch zur auffällig häufigen Arbeitsunfähigkeit.}

Ferner nimmt der Autor Anstoss an der merkwürdigen Zusammensetzung der Leitungsgruppe für das NFP53. Denn es ist sonderbar, dass die Leitungsgruppe für ein Forschungsprogramm, das den Bewegungsapparat betrifft, aus Experten der Geriatrie, Inneren Medizin, Neuropsychologie, Community Medicine, Sciencetechnique, Paraplegie, Nephrologie, Transplantationsimmunologie und aus dem Bundesamt für Gesundheit BAG besteht und diejenigen Ärzte fehlen,

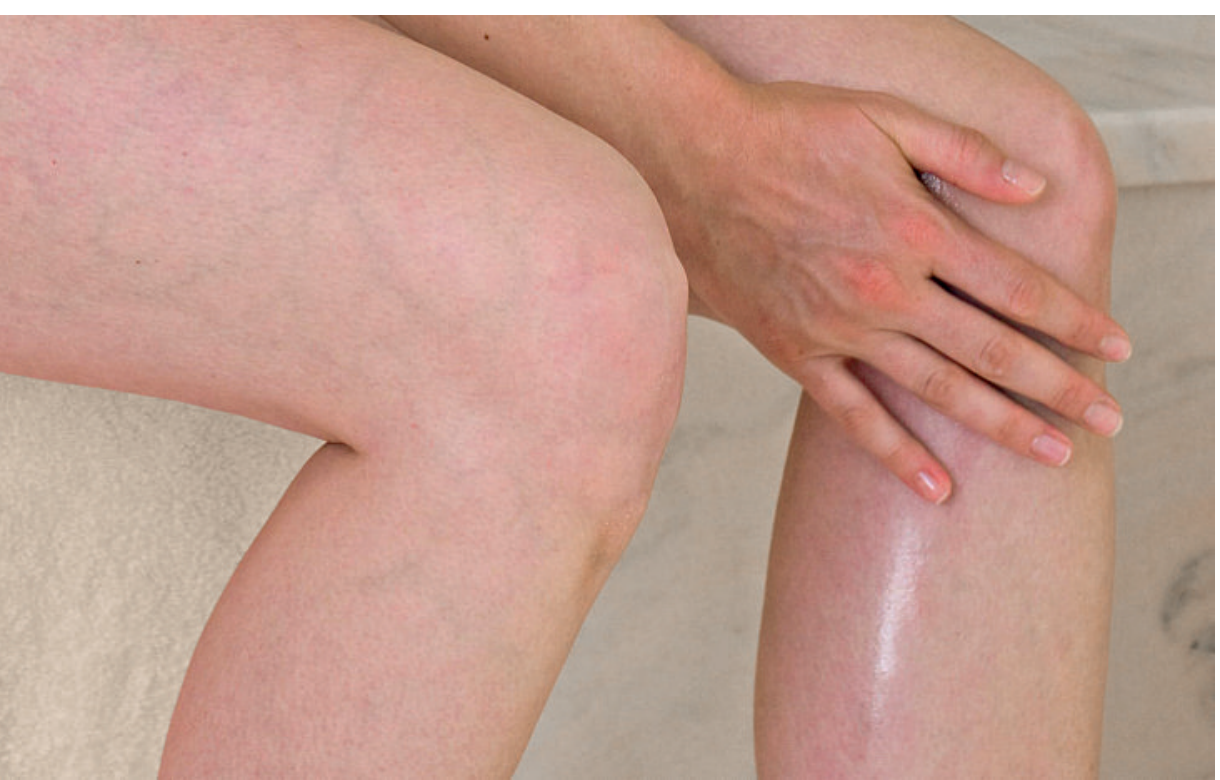

Die Behandlung von Schmerzen des Bewegungsapparates ist ein Tummelplatz für Therapeuten aller Art. stand, dass das NFP53 abgesehen vom Chondroitin als Placebo kaum Worte darüber verliert, dass der Bewegungsapparat Therapeuten aller Art als Tummelplatz dient. Der Verlauf vieler Beschwerden im Bereich des Bewegungsapparates mit spontanen Remissionen und beschwerdefreien Intervallen führt dazu, dass ausser bei bekannten Ausnahmen, vor allem bei Rückenbeschwerden die Behandlungsmethode eine untergeordnete oder sogar keine Rolle spielt $[2,3,4,5,6,7$, 8]. Dies erklärt die Vielfalt der als erfolgreich angepriesenen Behandlungsmethoden. Auf die Therapiefreudigkeit der vielen selbsternannten Experten, die sich um Patienten mit Beschwerden im Bereich des Bewegungsapparates kümmern und Therapien ohne Wirkungsnachweis gegen Bezahlung anbieten, wird im NFP53 nicht eingegangen. Es ist aber nicht belanglos, dass der Bewegungsapparat nicht nur für Chiropraktoren, sondern auch für zahlreiche andere Gesundheitspraktiker (Homöopathen, Akupunkteure, Neuraltherapeuten und Handaufleger) und neuerdings auch für die besonders spirituell anmutende Spiraldynamik zum einträglichen Tummelplatz geworden ist [4]. Diese «Experten» können gutgläubige Patienten davon überzeugen, während langer Zeit und auch prophylaktisch behandlungsbedürftig, arbeitsunfähig und sogar invalid zu sein.

Die Tatsache der spontanen Remissionstendenz mit beschwerdefreien Intervallen der Rückenbeschwerden steht im Widerspruch zur auffällig häufigen Arbeitsunfähigkeit und heute üblichen Behandlungsfreudigkeit bei vorübergehenden Rückenbeschwerden. Ich bin nicht der einzige orthopädische Chirurg, 
der bei der gutachterlichen Untersuchung und Beratung von Rentenbezügern über die Grosszügigkeit der Rentenzusprechungspraxis staunte.

\section{Zusammenfassung und Schlussfolgerung}

Das NFP53 hat auf die Mitarbeit von im Bereiche des Bewegungsapparates tätigen Medizinern und Chirurgen weitgehend verzichtet, hat die umfangreiche, in vielen Fachzeitschriften zu findende Literatur über den Bewegungsapparat zu wenig beachtet und ein Forschungsdefizit diagnostiziert. Das NFP53 brachte keine grundlegend neuen wichtigen Erkenntnisse. Die Behandlungspraktiken ohne Wirksamkeitsnachweis, die von zahlreichen Heilern an Patienten mit Beschwerden im Bereich des Bewegungsapparates ausgeübt werden, wurden kaum beachtet. Wenn diese Beurteilung auf weite Strecken zutrifft, stellt sich die Frage, ob das NFP53 den Einsatz von 12 Millionen Franken wert ist, eine Frage die sich bereits beim NFP34 und beim PEK stellte [12, 13].

Vorwiegend politisch motivierte Forschungsprojekte, die bei Berücksichtigung der existierenden Literatur mehrheitlich bereits bekannte Fakten erforschen, sind eigentlich überflüssig und kaum geeignet, dem Bundesrat und dem BAG mehr Sachverstand über den Bewegungsapparat zu vermitteln. Wahrscheinlich bin ich nicht der einzige Beobachter der medizinischen Szene und des Gesundheitsmarktes, der der Ansicht ist, dass politisch inszenierte Forschungsprogramme ausser Spesen, einer Belebung des Gesundheitsmarktes und der Erhaltung von einigen Arbeitsplätzen nichts bringen, auf das nicht verzichtet werden könnte. Solche nationalen Forschungsprogramme mögen wohl politicaly correct sein. Sie nehmen jedoch der seriösen Grundlagenforschung, die dringend auf finanzielle und personelle Förderung angewiesen ist, das dringend benötigte Geld weg.

\section{Literatur}

1 Leitungsgruppe des NFP53 Schweizerischer Nationalfonds (Hrsg.). Nationales Forschungsprogramm NFP53 «Muskuloskelettale Gesundheit chronische Schmerzen».

2 Salomon L et al. Appley's System of Orthopaedics and Fractures. New York: Arnold und Oxford University Press Inc.; 2001.

3 Allan DP, Waddell G. An historical perspective on low back pain and disability. Acta ortop Scand (Suppl); 1989.

4 Geiser M. Gedanken zur orthopädischen Chirurgie in der zweiten Hälfte des 20. Jahrhunderts. Schweiz Ärztezeitung. 200;81(39):2233-8.

5 Meade TW, Dyer S, Browne W, Townsend J, Frank AO. Low back pain of mechanical or;gin. Randomized comparison of chiropractic and hospital outpatient treatment. Br. Med. J. 1990;300:1431-7.

6 Geiser M. Epicondylitis humeri radialis. Schweiz Med Wschr; 1967;97:782-3.

7 Dickson RA. Spinal disorders and low back. J Bone and Joint Surg Br. 1999; 81:378-9.

8 Postachini F. Management of the herniation of the lumbar disc. J Bone and Joint Surg Br. 1999; 81;577-81.

9 Journal of Bone and Joint Surgery. Britische und Amerikanische Ausgabe.

10 Geiser M. Katamnese bei Rückenpatienten einer Infanterie-Rekrutenschule. Schweiz Med Wschr 1980; 110;1334-41.

11 Geiser M. Die Tragikomödie der Haltungsschäden. Schweiz Rundschau für Medizin PRAXIS. $1975 ; 64: 400-4$.

12 Geiser M. Das Ansehen nicht nur der FMH steht auf dem Spiel. Schweiz Ärztezeitung. 2005;86(8):470-2.

13 Stalder H. Das PEK - ein Epilog. Schweiz Ärztezeitung. 2010; 91(26/27):1074. 